\title{
Genomic and epigenomic predictors of response to guadecitabine in relapsed/ refractory acute myelogenous leukemia
}

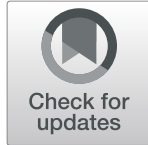

Woonbok Chung ${ }^{1,6^{*}} \mathbb{D}$, Andrew D. Kelly ${ }^{1}$, Patricia Kropf ${ }^{2}$, Henry Fung ${ }^{2}$, Jaroslav Jelinek ${ }^{1,6}$, Xiang Yao Su ${ }^{3}$, Gail J. Roboz ${ }^{4}$, Hagop M. Kantarjian', Mohammad Azab ${ }^{3}$ and Jean-Pierre J. Issa 1,6

\begin{abstract}
Background: Guadecitabine is a novel DNA methyltransferase (DNMT) inhibitor with improved pharmacokinetics and clinical activity in a subset of patients with relapsed/refractory acute myeloid leukemia ( $r / r \mathrm{AML})$, but identification of this subset remains difficult.

Methods: To search for biomarkers of response, we measured genome-wide DNA methylation, mutations of 54 genes, and expression of a panel of 7 genes in pre-treatment samples from 128 patients treated at therapeutic doses in a phase $1 / \|$ study.

Results: Response rate to guadecitabine was 17\% (2 complete remission (CR), 3 CR with incomplete blood count recovery (CRi), or CR with incomplete platelets recovery (CRp)) in the phase I component and 23\% (14 CR, 9 CRi/ (Rp) in phase II. There were no strong mutation or methylation predictors of response. Gene expression clustering defined a subset of patients ( 20\%) that had (i) high DNMT3B and low CDKN2B, CTCF, and CDA expression; (ii) enrichment for KRAS/NRAS mutations; (iii) frequent CpG island hypermethylation; (iv) low long interspersed nuclear element $1($ LINE-1) hypomethylation after treatment; and (v) resistance to guadecitabine in both phase I (response rate $0 \%$ vs. $33 \%, p=0.07$ ) and phase II components of the study (response rate $5 \%$ vs. $30 \%, p=0.02$ ). Multivariate analysis identified peripheral blood (PB) blasts and hemoglobin as predictors of response and cytogenetics, gene expression, RAS mutations, and hemoglobin as predictors of survival.
\end{abstract}

Conclusions: A subset of patients ( 20\%) with $\mathrm{r} / \mathrm{r}$ AML is unlikely to benefit from guadecitabine as a single agent. In the remaining $80 \%$, guadecitabine is a viable option with a median survival of 8 months and a 2-year survival rate of $21 \%$.

Trial registration: NCT01261312.

Keywords: AML, Guadecitabine, Mutations, DNA methylation inhibitor, Drug resistance, Gene expression

\section{Introduction}

Acute myeloid leukemia (AML) is a highly lethal hematopoietic malignancy characterized by poor longterm survival and relatively few somatic mutations compared to other cancer types [1,2]. Interestingly, the most commonly mutated genes in AML are enriched for

\footnotetext{
* Correspondence: wchung@coriell.org

${ }^{1}$ Fels Institute for Cancer Research and Molecular Biology, Temple University

School of Medicine, Philadelphia, PA, USA

${ }^{6}$ Present address: Coriell Institute for Medical Research, 403 Haddon Ave, Camden, NJ 08103, USA

Full list of author information is available at the end of the article
}

epigenetic regulators such as DNA methyltransferase 3A (DNMT3A), Tet methylcytosine dioxygenase 2 (TET2), and isocitrate dehydrogenase (IDH) $1 / 2$ [3]. In addition, DNA methylation in AML has been shown to harbor disruptions compared to normal blood [4], and various epigenetic signatures have been associated with differential chemotherapy response and prognosis [5]. At present, there are two hypomethylating agents (HMAs) - azacitidine and decitabine-which have been FDA approved for myelodysplastic syndromes (MDS) and are recommended for AML in patients who cannot tolerate intensive chemotherapy [6]. 
Current HMAs are considered low-intensity therapy in AML and function by blocking the activity of DNMT1, which in turn cannot copy DNA methylation across cell divisions. Although some improvements in remission and overall survival (OS) have been observed in MDS and AML, durable responses are rare, and one potential limitation is the low plasma half-life of these agents [7]. To address this issue, guadecitabine (formerly known as SGI-110) was developed to be resistant to degradation by cytidine deaminase (CDA) with a gradual release of active metabolite decitabine thereby exposing malignant cells to the active drug for longer. Guadecitabine is currently in several phase III clinical trials for AML and MDS after having shown promising phase I/II results [8-10].

Although HMAs such as guadecitabine induce remissions in AML, there are no validated biomarkers that can be used to select patients for therapy. This is especially relevant in relapsed AML, where the response rates are relatively low. In this study, we examined blood and/or bone marrow samples from relapsed/refractory AML ( $r / r$ AML) patients enrolled in phase I/II trials of guadecitabine for their DNA methylation status at baseline and following treatment, for baseline genetic mutations in a panel of 54 genes commonly mutated in hematopoietic malignancies, and for expression of a selected gene panel. Our data suggest that specific gene expression, DNA methylation, and mutational signatures may be associated with OS and therapy resistance in guadecitabine-treated patients.

\section{Materials and methods}

\section{Patients}

The phase I/II trials of guadecitabine were conducted under one study protocol and is registered with Clinical Trials.gov (NCT01261312). The patients' eligibility criteria in the phase I and the phase II stage were previously published $[8,10]$. The patients evaluated in this report are those with $\mathrm{r} / \mathrm{r}$ AML who were treated in the phase I stage on the $5 \times$ daily $\left(\geq 30 \mathrm{mg} / \mathrm{m}^{2}\right)$ or $3 \times$ weekly schedule $\left(\geq 60 \mathrm{mg} / \mathrm{m}^{2}\right)$ at therapeutic doses [8] and all those treated in phase II $\left(60\right.$ and $90 \mathrm{mg} / \mathrm{m}^{2}$ both in a 5day schedule and $60 \mathrm{mg} / \mathrm{m}^{2}$ in a 10-day schedule) [10] provided they had pre-treatment blood and/or bone marrow available for molecular analyses (Additional file 1 Table S1). Responses were evaluated by the IWG criteria [11]. Patients were considered responders if they had a composite complete response (CRc) including complete response (CR), $\mathrm{CRp}$ (CR with incomplete platelets recovery), or CRi (CR with incomplete blood count recovery). There were no statistically significant differences in the response rate or survival between the different doses/ schedules [10].

\section{Baseline gene expression analysis}

Whole-blood RNA was isolated and purified with the PAXgene Blood RNA Kit (Qiagen, Hilden, Germany) and QIAcube automated sample preparation system (Qiagen) by Covance Central Laboratory Services (Indianapolis, IN, USA), according to the manufacturer's instruction. Any contaminated DNA from RNA preparations was removed with TURBO DNA-free kit (Ambion, Carlsbad, CA, USA) before cDNA synthesis. First-strand cDNA was synthesized using High-Capacity cDNA Reverse Transcription Kits (Applied Biosystems, Grand Island, NY, USA). The quantitative expression of a panel of genes (CDA, CDKN2B (P15), CDKN1A (P21), DNMT1, DNMT3A, DNMT3B, and CCCTC-binding factor (CTCF)) at baseline was performed by TaqMan ${ }^{\circ}$ probe-based gene expression analysis by Applied Biosystems (Additional file 2 Table S2). These were selected because they are known epigenetic regulators (DNMT1, DNMT3A, DNMT3B, and CTCF), epigenetically regulated genes (P21 and P15), or determinant of decitabine levels (CDA). Human GAPDH was used as a normalization control in qPCR reactions. All qPCR reactions were run in triplicate and the values averaged. Negative controls where the reverse transcriptase enzyme was omitted were included in parallel to exclude the possibility of genomic DNA contamination. Relative target gene expression was represented by $\mathrm{dCT}$ (target gene $)=\mathrm{Ct}(\mathrm{GAPDH})-\mathrm{Ct}$ (target gene). Unsupervised hierarchical clustering analysis of baseline gene expression in the patients was performed by ArrayTrack (the National Center for Toxicological Research) using the Ward method [12].

\section{DNA methylation profiling}

Pre-treated whole-blood genomic DNA was purified with the QIAamp DNA Blood Mini Kit (Qiagen) and QIAcube automated sample preparation system (Qiagen) by Covance Central Laboratory Services. To analyze the genome-wide methylation profile, we used Digital Restriction Enzyme Analysis of Methylation (DREAM) $[13,14]$. Briefly, DREAM methylation analysis is a quantitative mapping of DNA methylation with high resolution on a genome-wide scale without bisulfite conversion. The method is based on sequential cuts of genomic DNA with a pair of neoschizomer endonucleases recognizing the same restriction site (CCCGGG) containing a CpG dinucleotide. The first enzyme, SmaI, cuts only at the unmethylated CpG sites and leaves blunt ends. The second enzyme, XmaI, is not blocked by methylation and leaves a short $5^{\prime}$ overhang. The enzymes thus generate distinct methylation-specific signatures at the ends of restriction DNA fragments. These are deciphered by next-generation sequencing. Methylation level at individual $\mathrm{CpG}$ sites is calculated as the 
ratio of sequencing reads with the methylated signature to the total number of reads mapping to the site. Using the DREAM method, we analyzed pre-treatment DNA methylation profiles of the $\mathrm{r} / \mathrm{r}$ AML patients $(n=116)$ from the phase I and II trials. Paired-end sequencing of 40 bases was performed on HiSeq 2500 (Illumina, San Diego, CA, USA) instrument at the Genomic Core Facility of Fox Chase Cancer Center (Philadelphia, PA, USA). These sequence data have been submitted to the GEO database under accession number GSE112838. We mapped the sequences to the human genome (hg19) and calculated the methylation at target sites as the fraction of reads with methylated signature. We included in the methylation analysis 17,793 CpG sites covered with at least 20 sequencing reads in at least $75 \%$ of samples. We imputed missing values ( $1.8 \%$ of total) by predictive mean matching [15] using the mice package in $R$ suite [16]. Unsupervised hierarchical clustering (Ward.D) and heatmap were performed by the pheatmap $R$ package. CpG site permutations were used to detect differentially methylated sites associated with response to guadecitabine. We used Ingenuity Pathway Analysis (Qiagen) for analyzing enriched canonical pathways of 121 genes with differentially methylated genes with $\mathrm{CR}$ and (separately) $\mathrm{CRc}$ in 116 patients. LINE-1 methylation dynamics were available on these patients from previous reports $[8,10]$.

\section{AML mutational profiles}

Pre-treatment blood or bone marrow-derived DNA was available for analysis from a total of 122 patients with AML enrolled on guadecitabine phase I/II trials. We performed mutation analysis by targeted highthroughput sequencing using the TruSight Myeloid Sequencing Panel (Illumina). The panel covers 54 genes commonly mutated in hematologic malignancies. FLT3-ITD was difficult to detect using this panel; we also detected this mutation by PCR followed by capillary electrophoresis (LabPMM, San Diego, CA, USA). Sequencing library preparation was performed according to the manufacturer's instruction. Paired-end sequencing of $2 \times 150$ bases was performed on the HiSeq 2500 instrument (Illumina), and reads were aligned to the human genome (hg19). Initial variants were called using the BaseSpace TrueSeq Amplicon app and BaseSpace Variant Studio 2.2 (Illumina). In addition, variants were required to have a minimum of 100 reads and a sequence quality score of at least 50. A minimum allelic fraction of $5 \%$ was set to identify somatic variants. Finally, we filtered the variants for presence in the COSMIC database (v78) with a hematopoietic malignancy association and absence from dbSNP (common variants) with a normal population frequency of $>1 \%$.

\section{Statistical analysis}

Fisher's exact test was used to compare the differences in the $\mathrm{CRc}$ or $\mathrm{CR}$ rates. Unpaired $t$ test or one-way ANOVA was used to compare the age, white blood cell (WBC) count, peripheral blood (PB) blast, and bone marrow (BM) blasts at screening in different (2 or 3 ) groups. We derived the maximum LINE-1 demethylation for patients during the first cycle of guadecitabine treatment. The mean maximum LINE-1 demethylation was compared between the groups using the MannWhitney test. Overall survival (OS) was measured as the time from the date of the first treatment to death (failure) or alive at last follow-up (censored). OS curves were estimated using the Kaplan-Meier method and compared between the groups using a log-rank test. To build a multivariate model of response to guadecitabine or OS by baseline expression, we derived a $z$-score of gene expression based on 4 genes (z4), obtained by $\mathrm{zCDA}+$ zP15 + zCTCF - zDNMT3B formula (based on gene expression clustering). Binary logistic regression and the Cox regression model were used for the univariate and the multivariate analysis, respectively, for 116 patients with mutational status and gene expression data to determine clinically meaningful differences in the achievement of CR, CRc, and OS. All $p$ values are two-sided, and $<0.05$ was considered significant. All the above statistical procedures were performed with GraphPad Prism (version 5.04) and SAS/STAT software (version 9.4).

\section{Results}

\section{Patients studied}

Additional file 3 Table S3 describes the demographic and clinical characteristics of the 29 patients on the phase I trial (17 of $25(68 \%))$ patients treated at therapeutic levels on the $5 \times$ daily schedule and 12 of 18 (67\%) patients on the $3 \times$ weekly schedule) and the 99 patients (of 103 patients treated or 96\%) on the phase II trials analyzed molecularly. The two groups were broadly similar with a CRc rate of $17 \%$ in phase I and $23 \%$ in phase II. Out of those 128 patients, we successfully obtained genome-wide methylation data in 116 (91\%), mutation data in 122 (95\%), and gene expression data in 122 (95\%) (Additional file 1: Table S1).

\section{Genome-wide DNA methylation analysis}

DREAM analysis of 17,793 sites in 116 patients identified 2774 hypervariable methylation sites (standard deviation $>10 \%$ ). Unsupervised hierarchical clustering of these sites divided the cases into three clusters (Fig. 1a). Cluster 1 contained all five samples of normal blood mononuclear cells analyzed and was therefore called "normal-like." Cluster 3 had more intense CpG island methylation reminiscent of the $\mathrm{CpG}$ island methylator phenotype (CIMP) $[5,17]$ and was therefore "CIMP-like," 

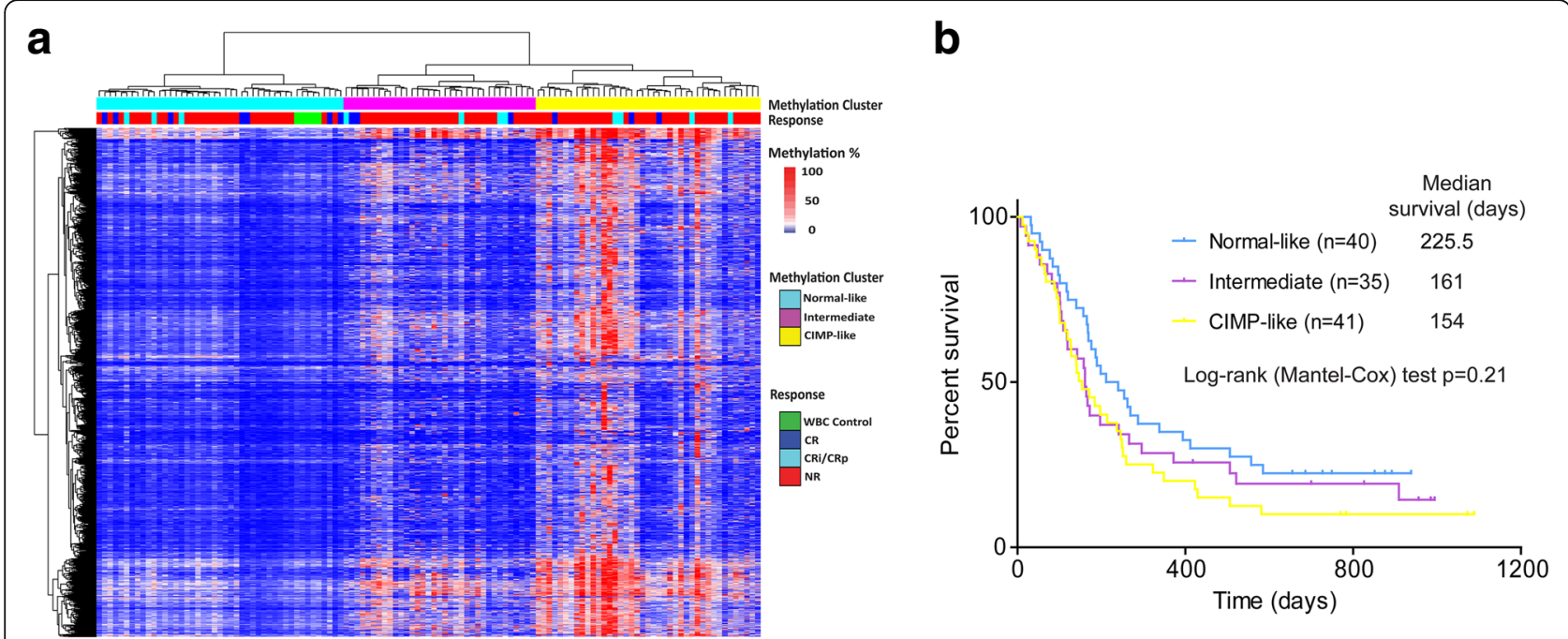

Fig. 1 DNA methylation and response to guadecitabine. a Unsupervised hierarchical clustering of $116 \mathrm{r} / \mathrm{r}$ AML patients based on 2774 hypervariable sites (standard deviation > 10\%) divided the cases into three clusters which were normal-like, CIMP-like, and intermediate methylation. b Kaplan-Meier survival analysis of 116 cases based on the clusters derived in $\mathbf{a}$. There was a trend for longer survival in the "normal-like" cluster, but this trend was not statistically significant $(p=0.21$ by log-rank test)

while cluster 2 was characterized by intermediate methylation. Clinical characteristics of the three clusters are shown in (Additional file 4: Table S4). There were trends for higher CR rate and longer survival (Additional file 4: Table S4 and Fig. 1b) in the "normal-like" cluster, but these trends were not statistically significant.

We next analyzed the correlations between methylation at individual $\mathrm{CpG}$ sites and $\mathrm{CR}$ or CRc rates in a two-step process: discovery in the 27 patients on the phase I trial and validation in the 87 patients on the phase II trial. There were only 29 sites out of 17,793 analyzed that were associated with CR in both phase I and II patients at a $p<0.05$ in both cohorts. We next combined data on all 116 patients and analyzed the correlations between methylation and CR in all. In this combined analysis, $879 \mathrm{CpG}$ sites showed statistically significant (empirical $p<0.05$ based on permutation testing) correlations with $\mathrm{CR}$ rate (and 459 sites with $\mathrm{CRc}$ rate). Among these, there was a significant enrichment for the sites in CTCF binding, predicted enhancer, and CpG islands (Additional file 5: Table S5). The sites included 121 genes with differentially methylated promoters, and Ingenuity Pathway Analysis of these is shown in (Additional file 6: Table S6). The most enriched pathway was the eicosanoid signaling pathway which is related to infection and inflammation. However, these data need validation in different sets of patients as a more stringent statistical test $(\mathrm{FDR}<0.01)$ revealed no differences between responders and non-responders.

\section{Correlations between mutation status and response}

We next queried for mutation status in a panel of 54 genes using the TruSight myeloid sequencing panel which analyzes a total of 338 exons (or 568 amplicons) by targeted deep sequencing. We successfully obtained data on a median aligned read of 94\% (range 48-98\%) in 122 patients (Fig. 2a). The median number of reads/ amplicons was 9125 (range 3646 to 25,050). Overall, the median mutation number was 1 per case (range 0 to 5 ). We compared the mutation frequencies for the 54 genes in the $122 \mathrm{r} / \mathrm{r}$ AML patients with those reported for treatment-naïve AML (tn-AML) in the TCGA [3] and found significant differences at ASXL1, FLT3 (-ITD), DNMT3A, and NPM1 gene by the Bonferroni correction $(p<0.0026$ to be significant after the Bonferroni adjustment of a major 19 genes comparison) (Additional file 7 : Table S7). None of the genes showed significant correlations between mutations and CRc (Additional file 8: Figure S1). When we analyzed the correlations with CR, none of the genes showed a significant correlation either (Fig. 2b). No gene showed synthetic lethality as evidenced by a high response rate among cases with mutations, though there were minor non-significant trends for TET2 (CR rate of 2/10 (20\%) in cases with mutations vs. $13 / 112(12 \%)$ in cases without, $p=0.35)$ and TP53 (CR rate of $2 / 9$ (22\%) in cases with mutations vs. 13/113 $(12 \%)$ in cases without, $p=0.31$ ). Multiple genes were mutated at a higher frequency in those patients who did not respond (Fig. 2b), though none reached statistical significance. These included KRAS, NRAS, IDH1, IDH2, and multiple others. As previously reported, KRAS and NRAS mutations were mutually exclusive, and there was a strong trend for RAS mutations ( $\mathrm{N}$ or $\mathrm{K}$ ) to be associated with resistance to guadecitabine (CR cases were seen in $0 / 22(0 \%)$ patients with RAS mutations 


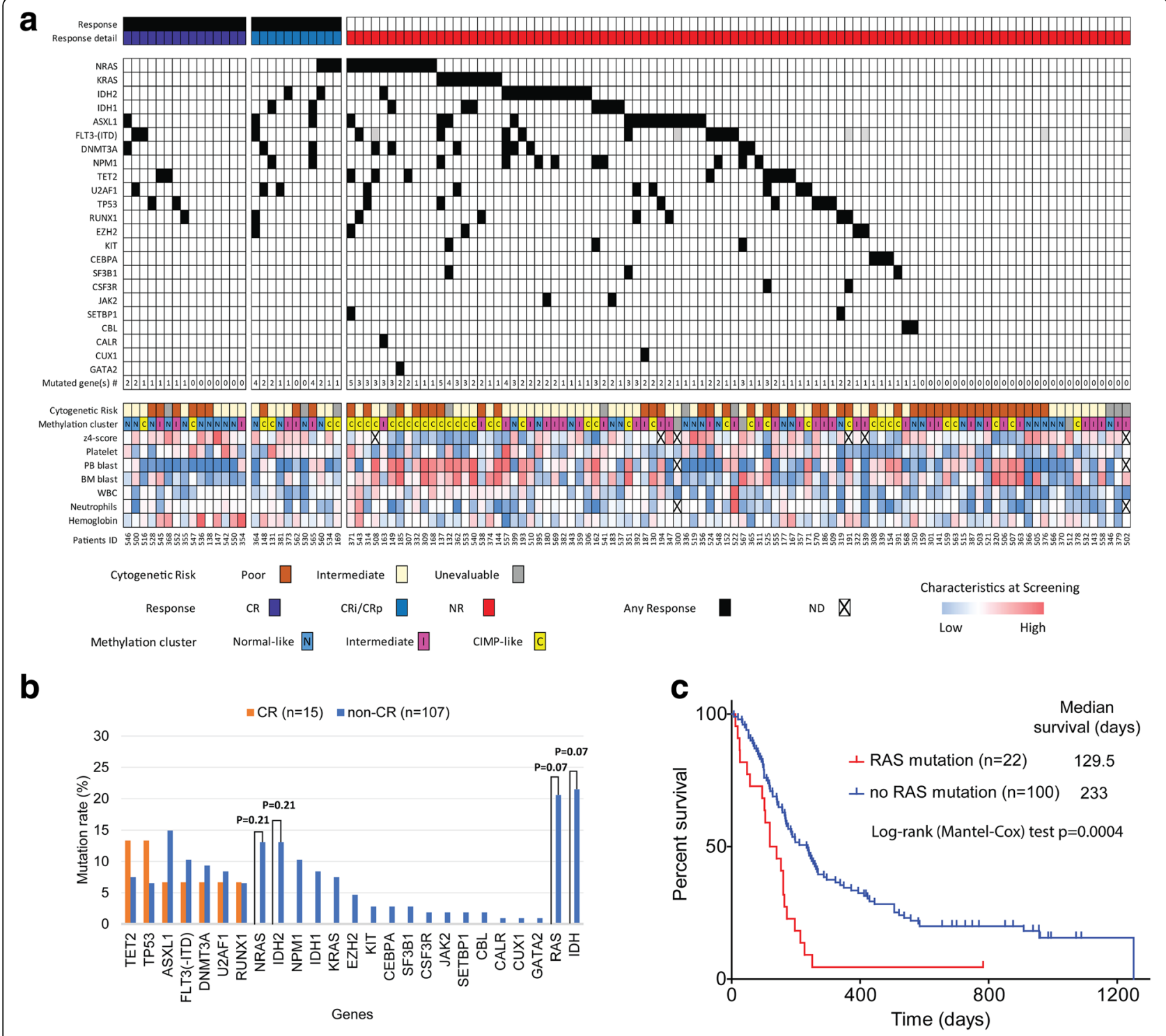

Fig. 2 Mutation spectrum and response to guadecitabine. a Association of mutation spectrum, clinical characteristics, and response to guadecitabine in $r / r$ AML $(n=122)$. Genomic mutation analysis was performed using the TruSight Myeloid Sequencing Panel (Illumina). The gene rows in the graph represent individual genomic lesions, the clinical characteristics row represent simplified clinical information, and the columns represent patients in the study. Black in the gene row indicates the presence of a specified mutation in a patient, and colors in the clinical characteristics row represent low (blue) to high (red). $\mathbf{b}$ Comparison of the mutation rate of CR vs. non-CR patients. None of the genes showed a significant correlation with $\mathrm{CR}$, but there was a strong trend for RAS mutations ( $\mathrm{N}$ or $\mathrm{K}$ ) to be associated with resistance to guadecitabine (CR were seen in 0/22 patients with RAS mutations compared to 15/100 patients without RAS mutations, $p=0.07)$. c Kaplan-Meier survival analysis stratified by RAS mutation status. The presence of RAS mutations was associated with a significantly worse survival ( $p=0.0004$ by log-rank test)

compared to $15 / 100(15 \%)$ patients without RAS mutations, $p=0.07$ ). The presence of RAS mutations was associated with a more aggressive clinical phenotype and more methylated (CIMP-like and intermediate) clusters (Table 1) and a significantly worse survival (Fig. 2c, median survival 129.5 days with RAS mutation vs. 233 days without RAS mutation, log-rank test $p=0.0004)$. IDH1/ 2 mutations also were associated with a trend for resistance to guadecitabine (CRs were seen in $0 / 23(0 \%)$ patients with IDH1/2 mutations compared to $15 / 99$ (15\%) patients without IDH1/2 mutations, $p=0.07)$. The presence of IDH2 mutation was associated with intermediate-risk cytogenetics (all 14 cases) and a significantly longer survival (Additional file 9: Figure S2, median survival 521 days with IDH2 mutation vs. 172 days without IDH2 mutation, log-rank test $p=0.03$ ). In the case of patients with IDH1 mutation, there was no difference in survival (median survival 240 days with IDH1 mutation vs. 176 days without IDH1 mutation, log-rank test $p=0.53$ ). 
Table 1 Mutation status in RAS and methylation status and clinical characteristics

\begin{tabular}{|c|c|c|c|}
\hline Characteristics & Mutation in RAS $(n=22)$ & No mutation in RAS $(n=100)$ & $p$ value \\
\hline Median age (range) & $54(29.1-81.7)$ & $62(23.4-86.1)$ & 0.14 \\
\hline Sex & & & 0.48 \\
\hline Male (\%) & $15(68 \%)$ & $59(59 \%)$ & \\
\hline Female (\%) & $7(32 \%)$ & $41(41 \%)$ & \\
\hline Cytogenetic risk & & & 1 \\
\hline Poor (\%) & $9(41 \%)$ & $42(42 \%)$ & \\
\hline Intermediate (\%) & $10(45 \%)$ & $50(50 \%)$ & \\
\hline Unevaluable (\%) & $3(14 \%)$ & $8(8 \%)$ & \\
\hline PB blasts at screening (\%), median (range) & $58(0-99)$ & $5.5(0-93)$ & $<0.0001$ \\
\hline BM blasts at screening (\%), median (range) & $49(18-95)$ & $31.1(2-94)$ & 0.0005 \\
\hline Platelet count $(\mathrm{K} / \mu \mathrm{L})$ at screening, median (range) & $31.5(7-230)$ & $36(2-342)$ & 0.44 \\
\hline Hemoglobin ( $\mathrm{g} / \mathrm{dL}$ ) at screening, median (range) & $9.4(7.2-12.6)$ & $9.3(6.0-14.4)$ & 0.96 \\
\hline WBC $(\mathrm{K} / \mu \mathrm{L})$ at screening, median (range) & $9.5(1.5-36.2)$ & $2.1(0.2-75.5)$ & 0.0007 \\
\hline LINE-1 maximum demethylation $\%$, mean $\pm \mathrm{SE}^{*}$ & $18.9 \pm 3.2$ & $24.3 \pm 1.3$ & 0.056 \\
\hline IDH mutation & $5(23 \%)$ & $18(18 \%)$ & 0.56 \\
\hline Methylation cluster & & & 0.0009 \\
\hline CIMP-like & $10(45 \%)$ & $31(31 \%)$ & \\
\hline Intermediate & $11(50 \%)$ & $24(24 \%)$ & \\
\hline Normal-like & 0 & $40(40 \%)$ & \\
\hline Unknown & $1(5 \%)$ & $5(5 \%)$ & \\
\hline \multicolumn{4}{|l|}{ Response } \\
\hline Complete CR rate (\%) & 0 & $15 / 100(15 \%)$ & 0.07 \\
\hline CRc rate (\%) & $3 / 22(14 \%)$ & $23 / 100(23 \%)$ & 0.4 \\
\hline Median survival days (range) & $129.5(12-783+)$ & $233(7-1088+)$ & 0.0004 \\
\hline 2-year survival rate & $1 / 22(5 \%)$ & 19/100 (19\%) & 0.12 \\
\hline
\end{tabular}

*Maximum LINE-1 demethylation for patients during the first cycle of guadecitabine treatment

\section{A gene expression predictor of resistance to guadecitabine}

We next examined the expression of a panel of 7 genes as potential predictors of response to guadecitabine. We used the patients on the phase I trial for discovery and the patients on the phase II trial for validation. In the 27 patients on the phase I trial, there were clear trends for correlations between expression and response (not shown), but most striking was evidence of co-regulation of these genes and an unsupervised hierarchical cluster analysis uncovered two groups of patients (Fig. 3a). Cluster R (resistant) was mainly characterized by low expression of DNMT1 and P15 and high expression of both DNMT3A and DNMT3B. Most strikingly, cluster $\mathrm{R}$ patients were resistant to guadecitabine, with $\mathrm{CRc}$ seen in $0 / 9(0 \%)$ patients compared to $5 / 18(28 \%)$ patients for cluster $\mathrm{S}$ (sensitive) $(p=0.14)$. Clinically, patients in cluster $\mathrm{R}$ had evidence of more aggressive AML with higher WBC, BM blasts, and PB blast counts (Additional file 10: Table S8). Based on this discovery cohort, we analyzed the same panel of genes in the 95 patients on the phase II trial and used hierarchical cluster analysis (Additional file 11: Table S9). As seen in Fig. 3b, a very similar dichotomy was observed in the validation cohort with a cluster of patients (cluster $R$ ) having high levels of DNMT3A and DNMT3B and low levels of DNMT1 and P15. These patients also showed resistance to guadecitabine (CRc seen in 1/21 (5\%) patients compared to $22 / 74$ (30\%) patients for cluster $\mathrm{S}$, $p=0.02$ ), thus confirming the initial data of the patients on the phase I trial. Patients in cluster $\mathrm{R}$ had worse survival after guadecitabine treatment (Additional file 11: Table S9, median survival 154 days in R cluster vs. 248 days in $\mathrm{S}$ cluster, log-rank test $p=0.0006$, and 2-year survival rate $0 / 21(0 \%)$ in $\mathrm{R}$ cluster vs. $18 / 74(24 \%)$ in $\mathrm{S}$ cluster, Fisher's exact test $p=0.01$ ). Hierarchical cluster analysis of all 122 patients in a combined phase I and II cohorts (Fig. 3c) refined the clusters; 27 patients (17\%) were in cluster $\mathrm{R}$ and had strikingly lower $\mathrm{CR}$ or CRc rates (CRc seen in 0/27 (0\%) patients compared to 28/95 (29\%) patients for cluster S, $p=0.0005)$. Patients in cluster $\mathrm{R}$ had higher WBC, BM blasts, and PB blast counts 


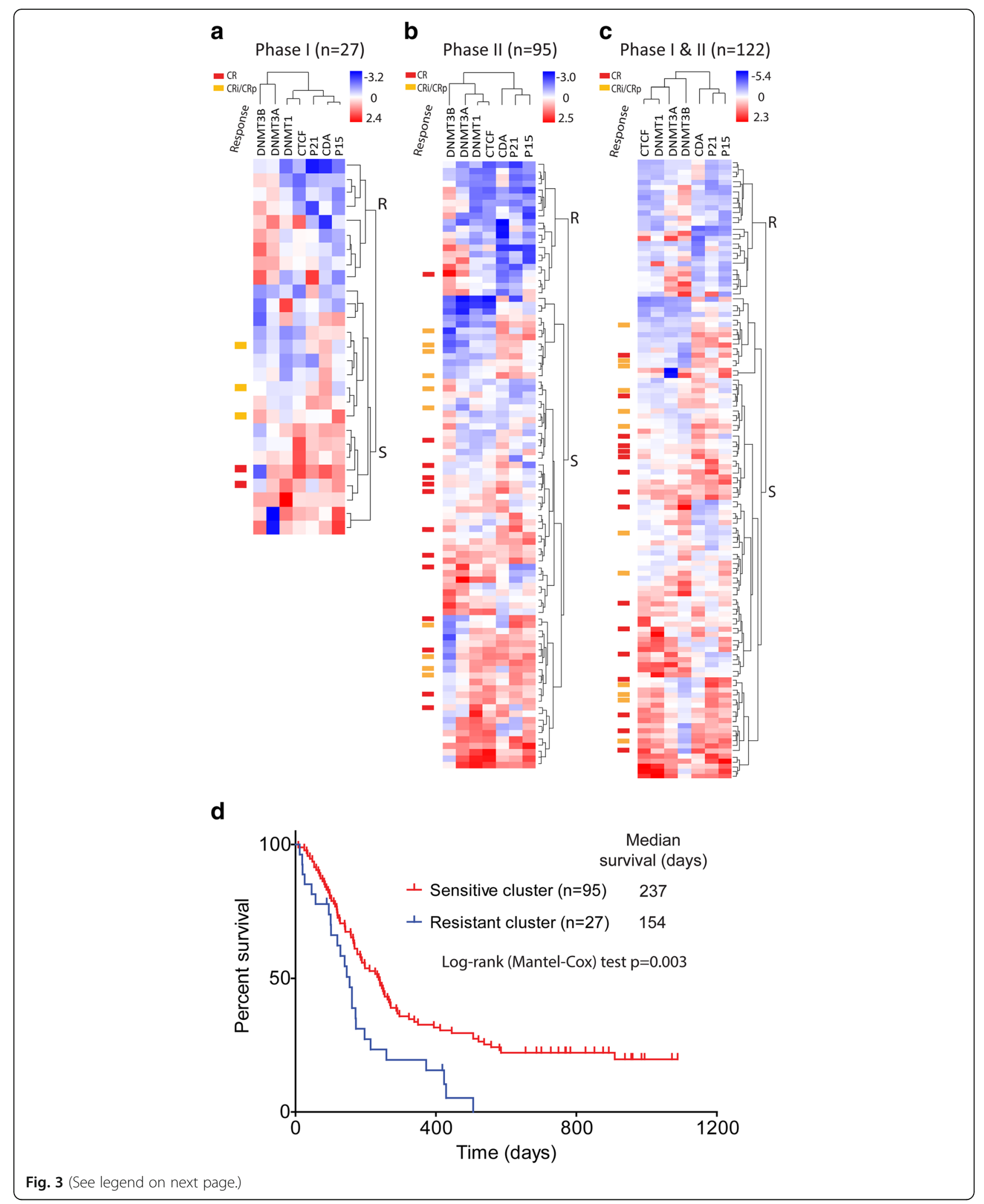


(See figure on previous page.)

Fig. 3 Selected gene expression profile and response to guadecitabine. a Unsupervised hierarchical clustering by baseline expression of the 7 gene panel grouped the phase I patients into two clusters $(n=27)$. Cluster $\mathrm{R}$ patients were clearly resistant to guadecitabine, with CRC seen in $0 / 9$ patients compared to $5 / 18$ patients for cluster $S(p=0.14)$. b Similar analyses of phase II patients $(n=95)$. These cluster $R$ patients also showed resistance to guadecitabine (CRc seen in $1 / 21$ patients compared to $22 / 74$ patients for cluster $S(p=0.02)$, thus confirming the initial data in phase I patients. $\mathbf{A}$ combined analysis of all 122 patients refined the clusters; 27 patients (17.2\%) were in cluster $R$ and had lower responses to guadecitabine (CRc seen in 0/27 patients compared to 28/95 patients for cluster $S(p=0.0005)$ ). $\mathbf{d}$ Kaplan-Meier survival analysis of all patients by the clusters derived in $\mathbf{c}$. Cluster $\mathrm{R}$ $(n=27)$ had a significantly worse survival after guadecitabine treatment ( $p=0.003$ by log-rank test)

(Table 2). Patients in cluster $\mathrm{R}$ also had worse survival after guadecitabine treatment (median survival 154 days in $\mathrm{R}$ cluster vs. 237 days in $\mathrm{S}$ cluster, logrank test $p=0.0028$, and 2 -year survival rate $0 / 27$ $(0 \%)$ in R cluster vs. 20/95 (21\%) in S cluster, Fisher's exact test $p=0.0065$ ) (Fig. $3 \mathrm{~d}$ and Table 2). We next used $z$-scores to derive quantitative surrogates for the gene expression clusters. A 4 gene $z$-score classifier (z4- score derived by zCDA + zP15 + zCTCF - zDNMT3B) discriminated well between clusters $\mathrm{R}$ and $\mathrm{S}$ and was also powerful at predicting CR or CRc. CRc rate was $2 / 38$ $(5 \%)$ at $\mathrm{z} 4$-score $<0$ and $26 / 84(31 \%)$ at $\mathrm{z} 4$-score $\geq 0$ (Fisher's exact test $p=0.0011$, Additional file 12: Figure S3). To compare how much these results are specific to AML patients, we performed a validation analysis in tn-AML cohort [3]. The univariate Cox

Table 2 Characteristics of patients by baseline gene expression cluster in combined phase I and phase II cohorts

\begin{tabular}{|c|c|c|c|}
\hline Characteristics & Resistant cluster $(n=27)$ & Sensitive cluster $(n=95)$ & $p$ value \\
\hline Median age (range) & $63(29.1-83.4)$ & $61(23.4-86.1)$ & 0.76 \\
\hline Sex & & & 0.37 \\
\hline Male (\%) & 19 (70\%) & $56(59 \%)$ & \\
\hline Female (\%) & $8(30 \%)$ & $39(41 \%)$ & \\
\hline Cytogenetic risk & & & 0.5 \\
\hline Poor (\%) & $14(52 \%)$ & $38(40 \%)$ & \\
\hline Intermediate (\%) & $12(44 \%)$ & $47(49 \%)$ & \\
\hline Unevaluable (\%) & $1(4 \%)$ & $10(11 \%)$ & \\
\hline PB blasts at screening (\%), median (range) & $72(10-99)$ & $4(0-86)$ & $<0.0001$ \\
\hline BM blasts at screening (\%), median (range) & $71(16-95)$ & $26(2-90)$ & $<0.0001$ \\
\hline Platelet count $(\mathrm{K} / \mu \mathrm{L})$ at screening, median (range) & $25(2-342)$ & $41(4-289)$ & 0.7 \\
\hline Hemoglobin (g/dL) at screening, median (range) & $9.3(6.0-11.7)$ & $9.2(6.5-14.4)$ & 0.33 \\
\hline WBC $(\mathrm{K} / \mu \mathrm{L})$ at screening, median (range) & $9.9(1-34)$ & $1.8(0-76)$ & 0.0005 \\
\hline LINE-1 maximum demethylation $\%$, mean \pm SE & $15.4 \pm 2.3$ & $26.3 \pm 1.4$ & 0.0002 \\
\hline RAS mutation & $12 / 27(44 \%)$ & $9 / 89(10 \%)$ & 0.0002 \\
\hline IDH mutation & $6 / 27(22 \%)$ & $17 / 89(19 \%)$ & 0.78 \\
\hline z4-score mean $\pm S D$ & $-2.4 \pm 1.26$ & $1.90 \pm 1.85$ & $<0.0001$ \\
\hline Methylation cluster & & & $<0.0001$ \\
\hline CIMP-like & $18(67 \%)$ & $23(24 \%)$ & \\
\hline Intermediate & $9(33 \%)$ & $23(24 \%)$ & \\
\hline Normal-like & 0 & $38(40 \%)$ & \\
\hline Unknown & 0 & $11(12 \%)$ & \\
\hline \multicolumn{4}{|l|}{ Response } \\
\hline CR rate $(\%)$ & 0 & $16 / 95(17 \%)$ & 0.02 \\
\hline CRc rate (\%) & 0 & $28 / 95(29 \%)$ & 0.0005 \\
\hline Median survival days (range) & $154(12-506)$ & $237(8-1088+)$ & 0.003 \\
\hline 2-year survival rate & 0 & 20/95 (21\%) & 0.0065 \\
\hline
\end{tabular}


regression analysis showed high expression of DNMT3B was associated with worse OS (hazard ratio $(\mathrm{HR})=1.26,95 \%$ CI $1.07-1.49, p=0.005)$, but CTCF, P15 and CDA were not.

\section{An integrated analysis of resistance to guadecitabine} Of all the molecular studies described earlier, gene expression of a panel of 7 genes and the presence of RAS mutations were the most promising predictive markers, with both being associated with resistance to guadecitabine. As shown in Tables 1 and 2, these two molecular events were associated. Patients in the gene expression "resistance" cluster were characterized by a more aggressive clinical course (higher WBC, BM blasts, and PB blast counts) and had a higher incidence of RAS mutations. Interestingly, DNA methylation clusters showing CIMP-like and intermediate patterns were also enriched in the resistance cluster $(p<0.0001)$ while cytogenetics showed no differences. As expected, z4-scores of gene expression were dramatically different between clusters $\mathrm{R}$ and $\mathrm{S}$ (Table 2), and they were inversely correlated with WBC count $(r=-0.24)$, PB blast count $(r=-0.86)$, and BM blast count $(r=-0.62)$. Patients with RAS mutations had a lower z4-score $(-0.99$ in case of RAS mutations vs. 1.39 in case of RAS wild type, $p<0.0001$ ) but no significant differences in cytogenetics risk.

Towards an integrated model, we started with univariate logistic regression models for response (either CRc or CR). PB blasts, BM blasts, z4-score, and hemoglobin count were all significant, both $\mathrm{CR}$ and $\mathrm{CRc}$ at $p<0.01$ (Additional file 13: Figure S4 and Additional file 14: Figure S5). In a multivariate model, hemoglobin level and PB blasts were significant predictors of CRc while hemoglobin level and z4-score were significant predictors of CR. The logistic regression model for CR could not be fitted for resistance clusters, IDH, or RAS mutation because there were no CR cases that were positive. We next examined survival. In a univariate Cox regression model, PB blasts, z4-score, BM blasts, RAS mutation, cytogenetic risk, and cluster of resistance were all significant at $p<0.01$ (Fig. 4a and Additional file 15: Figure S6). In a multivariate Cox analysis, cytogenetic risk and the presence of RAS mutation were significant predictors of worse survival, while a higher z4-score and hemoglobin counts were significant predictors of longer survival (Fig. 4b).

Thus, a subset of patients with $\mathrm{r} / \mathrm{r}$ AML characterized by a unique gene expression pattern, RAS mutations, high levels of $\mathrm{CpG}$ island methylation, high BM blasts, and high PB blasts is associated with poor response and poor survival after guadecitabine treatment. In multivariate analyses, a high hemoglobin level and low PB blasts were the best predictors of $\mathrm{CRc}$, while gene expression (z4-score), cytogenetics, hemoglobin, and RAS mutations were all predictors of OS.

\section{Discussion}

Guadecitabine (SGI-110) is a novel hypomethylating dinucleotide of decitabine and deoxyguanosine that is resistant to degradation by cytidine deaminase and results in prolonged in vivo exposure to its active moiety decitabine [8]. This differential pharmacokinetic profile offers the potential of improved biological and clinical activity and safety over currently available first-generation drugs such as azacitidine and decitabine. To develop genomic predictors of response to guadecitabine for $\mathrm{r} / \mathrm{r}$ AML patients, we investigated hematopoietic cell samples from the patients enrolled in phase I/II trials of guadecitabine for their DNA methylation status, major genetic mutations, and a panel of gene expression at baseline. Our data suggested that there were no strong genetic, epigenetic, or gene expression predictors of CR or CRc to guadecitabine in $\mathrm{r} / \mathrm{r}$ AML. From a DNA methylation perspective, the most enriched pathway of differentially methylated genes in responders to guadecitabine was the eicosanoid signaling pathway, possibly implying a role for inflammation and immunity [18] in these responses. However, this study suffers from potential overfitting effects and the data need to be validated in different population cohorts. The lack of correlation between global patterns of baseline DNA methylation and response is consistent with previous studies [19] and reflects the multifactorial nature of sensitivity to DNMT inhibitors [20].

From a genetic change perspective, the only genes with strong trends for (inverse) correlation with response were RAS (KRAS and NRAS) and IDH2. The data on IDH2 were surprising given that these patients have a strong hypermethylation phenotype [5, 21]; it is possible that the lack of TET function in these cases compromises the ability to stably demethylate and respond to HMAs. Interestingly, in a trial of an IDH2 inhibitor for $\mathrm{r} / \mathrm{r}$ AML patients with IDH2 mutations, patients with NRAS mutations also had a worse prognosis [22]. The lack of an effect of TP53 mutations on response is different than what was observed with decitabine [23] and could reflect the fact that the population of patients treated here had relapsed/refractory disease. Of note, other studies have not found a correlation between TP53 mutations and response to HMAs [24]. Interestingly, the best molecular predictor of OS included gene expression of DNMT3B and CTCF which are wellknown epigenetic regulators. CTCF is a zinc-finger DNA-binding protein that functions as transcriptional repressor or activator, as an insulator that can block the ability of enhancers to activate promoters, and as a threedimensional (3D) chromatin organization. CTCF binding is determined by DNA sequence, methylation, and 
a

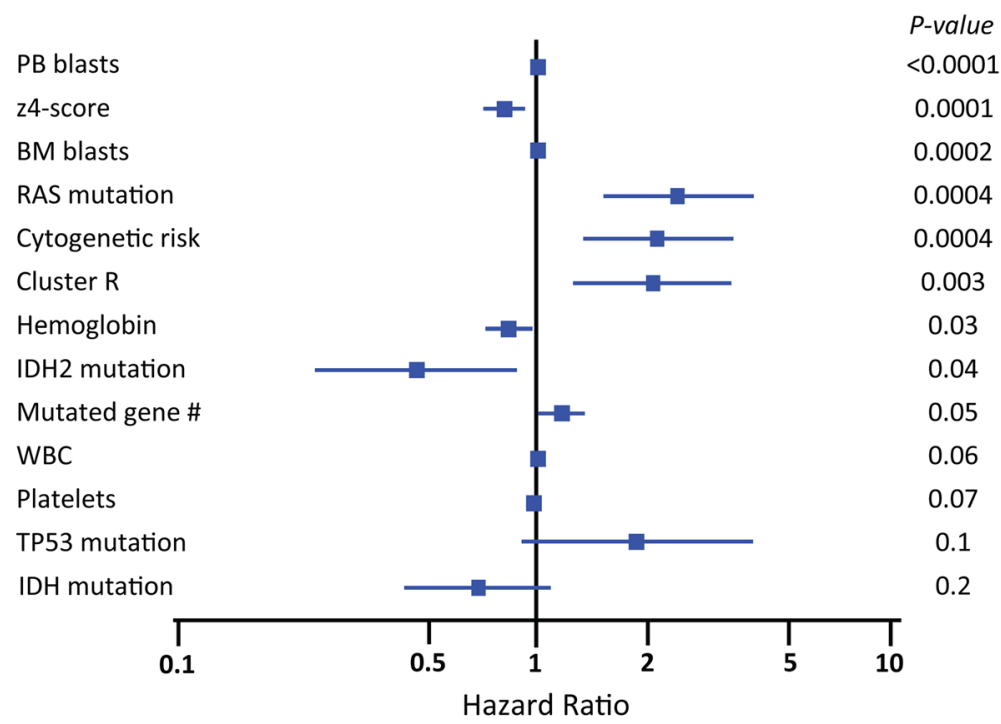

b

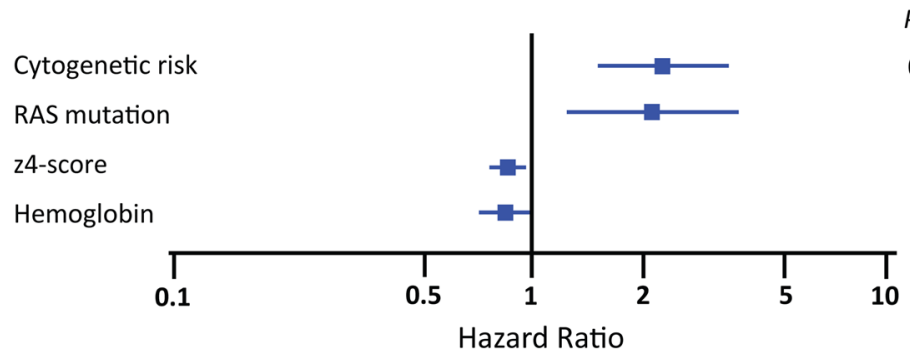

P-value

$<0.0001$

0.0002

0.0004

0.0004

0.003

0.03

0.04

0.05

0.06

0.07

0.1

0.2
0.0003

0.01

0.01

0.04

Fig. 4 Univariate and multivariate COX regression to study survival after guadecitabine. a In univariate COX regression analyses, significant factors in univariate analyses were PB blasts ( $\mathrm{HR}=1.01,95 \% \mathrm{Cl} 1.01-1.02, p<0.0001)$, z4-score ( $\mathrm{HR}=0.85,95 \% \mathrm{Cl} 0.78-0.91, p<0.0001)$, BM blasts ( $\mathrm{HR}=1.02$, $95 \% \mathrm{Cl} 1.01-1.02, p=0.0002)$, RAS mutation ( $\mathrm{HR}=2.53,95 \% \mathrm{Cl} 1.45-3.92, p=0.0004)$, cytogenetic risk $(\mathrm{HR}=2.18,95 \% \mathrm{Cl} 1.41-3.17, p=0.0004)$, cluster $\mathrm{R}$ $(\mathrm{HR}=2.03,95 \% \mathrm{Cl} 1.26-3.17, p=0.003)$, hemoglobin value $(\mathrm{HR}=0.86,95 \% \mathrm{Cl} 0.75-0.99, p=0.03), \mathrm{IDH} 2$ mutation $(\mathrm{HR}=0.47,95 \% \mathrm{Cl} 0.23-0.98, p=$ 0.044), and mutated gene number ( $\mathrm{HR}=1.20,95 \% \mathrm{Cl} 1.00-1.43, p=0.048)$. b In a multivariate analysis by backward regression, cytogenetic risk $(H R=2.25,95 \% \mathrm{Cl} 1.46-3.48, p=0.0003)$, $z 4$-score $(H R=0.89,95 \% \mathrm{Cl} 0.81-0.97, p=0.01)$, the presence of RAS mutation (HR=2.12, 95\% $\mathrm{Cl} 1.19-3.76, p=0.01)$, and hemoglobin value ( $\mathrm{HR}=0.86,95 \% \mathrm{Cl} 0.74-0.99, p=0.04)$ were significant predictors of survival

nucleosome occupancy [25]. DNMT3B and DNMT3A are responsible for establishing de novo DNA methylation patterns. DNMT3B and DNMT1 levels had opposite effects on the outcome after guadecitabine. From our validation analysis in the tn-AML cohort [3], we confirmed high expression of DNMT3B was associated with worse OS. Similar observations of worse clinical outcomes with high expression of DNMT3B were reported in de novo AML [26], pediatric AML [27], and older adult patients with cytogenetically normal AML [28]; this observation needs to be confirmed in genome-wide studies. In a mouse study, Dnmt3b, but not Dnmt3a, is upregulated following Dnmt1 deletion, and Dnmt3b is required for the survival of intestinal epithelium-specific Dnmt1-mutant mice [29]. In established HMA-resistant cell lines from a human monocytic leukemia cell line (MOLM-13), protein levels of DNMT3B were found upregulated compared to parental cell line and mRNA expression of DNMT1 and DNMT3A in HMA-resistant cell lines decreased compared to parental cell line [30]. In MDS-derived cell lines, the protein expressions of DNMT1 and DNMT3A, but not DNMT3B, tend to decrease in the presence of HMAs [31]. Additionally, cancer cell lines harboring DNMT3B gene amplification are less sensitive to the decrease in cell viability caused by HMAs [32]. Taken together, DNMT1, DNMT3A, and DNMT3B have different sensitivity to HMAs, and DNMT1 and DNMT3A, rather than DNMT3B, are the major targets of HMAs. And upregulation of DNMT3B could be a signature of resistance to HMAs. This baseline gene expression signature would be reflecting higher heterogeneity of HMA-resistant AML cells.

An integrated analysis identified a group of patients showing clear resistance to guadecitabine. They had a baseline expression signature of high-level expression of 
DNMT3B and low-level expression of P15, CTCF, and CDA; enrichment for KRAS or NRAS mutations; and a high degree of $\mathrm{CpG}$ island methylation. Clinically, these patients had higher bone marrow and peripheral blast counts, and interestingly, they had a significantly lower LINE-1 hypomethylation induction. Multivariate analysis also identified higher hemoglobin levels as a predictor of response and longer survival. It was reported that a hemoglobin value of at least $10 \mathrm{~g} / \mathrm{dL}$ was associated with longer OS in patients with myelodysplastic syndromes treated with decitabine [33]. It is apparent therefore that the most aggressive relapsed/refractory AMLs are relatively refractory to single-agent guadecitabine, possibly because the pace of disease evolution overwhelms the ability of a slow-acting approach such as HMAs. If these patients could be identified prospectively, they may benefit from alternate approaches such as cytotoxic therapy, HMAs in combination with other therapies, or possibly a higher dose of HMAs. Indeed, it was reported that high-dose cytarabine treatment improves the responses in AML patients with RAS mutation [34]. Also, the phase III study of guadecitabine in $\mathrm{r} / \mathrm{r}$ AML patients is using the 10-day regimen for up to 2 cycles with an allowance starting the second cycle earlier than 4 weeks in an attempt to provide a more myelosuppressive regimen upfront.

\section{Conclusions}

We identified a subset of patients ( 20\% based on gene expression) with relapsed or refractory AML who are unlikely to show CR or CRc after a single-agent guadecitabine therapy. In the remaining $80 \%$ of patients without this poor prognosis signature, guadecitabine is an excellent option in $\mathrm{r} / \mathrm{r}$ AML with a median survival of $\sim 8$ months and a 2 -year survival rate of $21 \%$. In the future, gene expression analysis and RAS mutation measurements may help in the development of effective personalized treatment strategies for patients with $\mathrm{r} / \mathrm{r}$ AML.

\section{Additional files}

Additional file 1: Table S1. Summary of studies reported in this paper (XLSX 8 kb)

Additional file 2: Table S2. List of TaqMan gene expression assays* used in this study. (XLSX $8 \mathrm{~kb}$ )

Additional file 3: Table S3. Demographic and baseline characteristics of the analyzed $r / r$ AML patients in phase I and II (XLSX $11 \mathrm{~kb}$ )

Additional file 4: Table S4. Clinical characteristics of relapsed/refractory AML patients at baseline, survival, and response in the CGI methylation clusters (XLSX $11 \mathrm{~kb}$ )

Additional file 5: Table S5. Locations of 879 differentially methylated Smal CpG sites correlated with complete response (CR) in the methylation analysis of a total of 17,793 CpG sites ( $\geq 20$ reads) by DREAM analysis (empirical $p<0.05$ based on permutation testing). (XLSX $10 \mathrm{~kb}$ )
Additional file 6: Table S6. Canonical pathway analysis (Ingenuity Pathway Analysis) of 121 genes with differentially methylated promoters from an analysis of 17,793 CpG sites ( $\geq 20$ reads). (XLSX 8 kb)

Additional file 7: Table S7. Comparison of the major 19 mutated genes in this study and TCGA tn-AML $(n=200)$. $(p<0.0026$ is significant after the Bonferroni adjustment) (XLSX $10 \mathrm{~kb}$ )

Additional file 8: Figure S1. Comparison of the mutation rate of composite complete response (CRc) to guadecitabine vs. non-response patients. None of the genes showed a significant correlation with CRc, but there was a trend for KRAS mutations to be associated with resist ance to guadecitabine (CRc were seen in 0/26 patients with KRAS muta tions compared to $8 / 96$ patients without KRAS mutations, $p=0.2$ ). (TIF $1237 \mathrm{~kb})$

Additional file 9: Figure S2. Kaplan-Meier survival analysis stratified by IDH2 mutation status. The presence of IDH2 mutations was associated with a significantly better survival (median survival 521 days with IDH2 mutation vs. 171.5 days without IDH2 mutation, log-rank test $p=0.03$ ). (TIF 1167 kb)

Additional file 10: Table S8. Characteristics of $r / r$ AML patients by baseline gene expression cluster in phase I (XLSX $11 \mathrm{~kb}$ )

Additional file 11: Table S9. Characteristics of $r / r$ AML patients by baseline gene expression cluster in phase II (XLSX $11 \mathrm{~kb})$

Additional file 12: Figure S3. Waterfall plot of z4-score and response. We used $z$-scores to derive quantitative surrogates for the gene expres sion clusters. A 4 gene $z$-score classifier (z4-score derived by zCDA + zP15 + zCTCF - zDNMT3B) was powerful at predicting CR or CRc. CRc rate was $2 / 38(5 \%)$ at $z 4$-score $<0$ and $26 / 84(31 \%)$ at $z 4$-score $\geq 0$ (Fisher's exact test $p=0.0011)$. (TIF $1032 \mathrm{~kb}$ )

Additional file 13: Figure S4. Univariate and multivariate logistic regression of composite complete response (CRC) to guadecitabine. In univariate logistic regression analyses of $C R c$, significant factors were hemoglobin value (odds ratio $(\mathrm{OR})=1.61,95 \% \mathrm{Cl} 1.18-2.20, p=0.003)$, $\mathrm{z} 4$ -score $(\mathrm{OR}=1.41,95 \% \mathrm{Cl} 1.13-1.76, p=0.003), \mathrm{PB}$ blasts $(\mathrm{OR}=0.96,95 \%$ $\mathrm{Cl} 0.94-0.99, p=0.005)$, and BM blasts $(\mathrm{OR}=0.97,95 \% \mathrm{Cl} 0.95-0.99, p=$ 0.006). In a multivariate analysis by backward regression, hemoglobin value $(\mathrm{OR}=1.56,95 \% \mathrm{Cl} 1.14-2.15, p=0.006)$ and $\mathrm{PB}$ blasts $(\mathrm{OR}=0.96$, $95 \% \mathrm{Cl} 0.93-0.99, p=0.007$ ) were significant predictors of response. (TIF $1009 \mathrm{~kb})$

Additional file 14: Figure S5. Univariate and multivariate logistic regression of complete response (CR) to guadecitabine. In univariate logistic regression analyses of $C R$, significant factors were hemoglobin value $(\mathrm{OR}=1.84,95 \% \mathrm{Cl} 1.27-2.5, p=0.001)$, z4-score $(\mathrm{OR}=1.48,95 \% \mathrm{Cl}$ $1.11-1.98, p=0.008)$, BM blasts (OR=0.97, 95\% Cl 0.94-0.996, $p=0.03)$, and PB blasts $(\mathrm{OR}=0.95,95 \% \mathrm{Cl} 0.90-0.995, p=0.03)$. In a multivariate analysis, hemoglobin value $(\mathrm{OR}=1.70,95 \% \mathrm{Cl} 1.18-2.46, p=0.005)$ and $\mathrm{z} 4$-score $(\mathrm{OR}=1.43,95 \% \mathrm{Cl} 1.04-1.95, p=0.03)$ were significant predictors of response. (TIF $948 \mathrm{~kb}$ )

Additional file 15: Figure S6. Univariate COX regression of z4 component genes. In univariate COX regression analyses of z4 component genes, the significant factors in univariate analyses were zCTCF $(H R=0.66,95 \% \mathrm{Cl} 0.52-0.84, p=0.0006), z D N M T 3 B(H R=1.52,95 \%$ Cl 1.17-1.98, $p=0.002), z C D A(H R=0.77,95 \% \mathrm{Cl} 0.62-0.96, p=0.02)$, and zP15 (HR=0.82, 95\% Cl 0.66-1.02, $p=0.08)$. (TIF $1079 \mathrm{~kb})$

\section{Abbreviation}

BM: Bone marrow; CDA: Cytidine deaminase; CIMP: CpG island methylator phenotype; CR: Complete remission; CRc: Composite complete response; CRi: CR with incomplete blood count recovery; CRp: CR with incomplete platelets recovery; CTCF: CCCTC-binding factor; DNMT: DNA methyltransferase; DREAM: Digital Restriction Enzyme Analysis of Methylation; HMA: Hypomethylating agent; IDH: Isocitrate dehydrogenase; LINE-1: Long interspersed nuclear element 1; MDS: Myelodysplastic syndromes; OS: Overall survival; P15: CDKN2B Cyclin-dependent kinase inhibitor 2B; P21: CDKN1A Cyclin-dependent kinase inhibitor 1; PB: Peripheral blood; r/r AML: Relapsed/ refractory acute myeloid leukemia; TET: Tet methylcytosine dioxygenase; tn -AML: Treatment-naïve acute myeloid leukemia; WBC: White blood cell 


\section{Authors' contributions}

J-PJI and MA have made substantial contributions to the conception, design, and study supervision. WC and JJ performed the scientific experiments and interpretation of the data. PK, HF, GR, HMK, J-PJI, XYS, and MA have contrib uted to the treatment of patients on protocol and acquisition of the data. WC, ADK, JJ, XYS, MA, and J-PJI have contributed to the analysis and inter pretation of the data. WC and XYS have contributed to the administrative, technical, and material support. WC and J-PJI prepared and review the exper iments, figures, and tables. All authors have been involved in the drafting of the manuscript and approval of the final manuscript.

\section{Funding}

This work was supported by the National Institutes of Health grants R01CA158112 and P50CA100632 and by a grant from Astex Pharmaceuticals Inc. J-PJl is an American Cancer Society Clinical Research professor supported by a generous gift from the FM Kirby Foundation.

\section{Availability of data and materials}

The datasets generated and analyzed during the current study are available from the corresponding author on reasonable request. In addition, the DNA methylation profiles discussed in this publication have been deposited in NCBI's Gene Expression Omnibus database under accession number GSE112838.

\section{Ethics approval and consent to participate}

The study protocol was approved by each institutional review boards at every participating site and was conducted in accordance with the Declaration of Helsinki, Good Clinical Practice, and all local and federal regulatory guidelines. A parent or legal guardian provided written informed consent, with patient assent as appropriate according to institutional requirements.

\section{Consent for publication}

Not applicable

\section{Competing interests}

XYS and MA are employees of Astex Pharmaceuticals. GJR and J-PJI received consulting fees from an advisory committee, and J-PJ received research funding from Astex Pharmaceuticals. WC, ADK, PK, HF, JJ, and HMK declare that they have no competing interests.

\section{Author details}

'Fels Institute for Cancer Research and Molecular Biology, Temple University School of Medicine, Philadelphia, PA, USA. ${ }^{2}$ Fox Chase Cancer Center, Temple Health, Philadelphia, PA, USA. ${ }^{3}$ Astex Pharmaceuticals Inc., Pleasanton, CA, USA. ${ }^{4}$ Weill Cornell Medicine, Division of Hematology and Oncology, The New York Presbyterian Hospital, New York, NY, USA. ${ }^{5}$ MD Anderson Cancer Center, Houston, TX, USA. ${ }^{6}$ Present address: Coriell Institute for Medical Research, 403 Haddon Ave, Camden, NJ 08103, USA.

\section{Received: 27 March 2019 Accepted: 12 July 2019}

\section{Published online: 22 July 2019}

\section{References}

1. Khwaja A, et al. Acute myeloid leukaemia. Nat Rev Dis Primers. 2016;2:16010.

2. Bullinger L, Döhner K, Döhner H. Genomics of acute myeloid leukemia diagnosis and pathways. J Clin Oncol. 2017;35(9):934-46.

3. Ley TJ, et al. Genomic and epigenomic landscapes of adult de novo acute myeloid leukemia. N Engl J Med. 2013;368(22):2059-74.

4. Toyota M, et al. Methylation profiling in acute myeloid leukemia. Blood. 2001; 97(9):2823-9.

5. Kelly $A D$, et al. A CpG island methylator phenotype in acute myeloid leukemia independent of IDH mutations and associated with a favorable outcome. Leukemia. 2017;31(10):2011-9.

6. Sato T, Issa JJ, Kropf P. DNA hypomethylating drugs in cancer therapy. Cold Spring Harb Perspect Med. 2017;7:a026948.

7. Issa JP, Kantarjian HM. Targeting DNA methylation. Clin Cancer Res. 2009; 15(12):3938-46.

8. Issa JP, et al. Safety and tolerability of guadecitabine (SGI-110) in patients with myelodysplastic syndrome and acute myeloid leukaemia: a multicentre, randomised, dose-escalation phase 1 study. Lancet Oncol. 2015; 16(9):1099-110.
9. Kantarjian HM, et al. Guadecitabine (SGI-110) in treatment-naive patients with acute myeloid leukaemia: phase 2 results from a multicentre, randomised, phase 1/2 trial. Lancet Oncol. 2017;18(10):1317-26.

10. Roboz GJ, et al. Dose, schedule, safety, and efficacy of guadecitabine in relapsed or refractory acute myeloid leukemia. Cancer. 2018;124(2):325-34

11. Cheson $\mathrm{BD}$, et al. Revised recommendations of the international working group for diagnosis, standardization of response criteria, treatment outcomes, and reporting standards for therapeutic trials in acute myeloid leukemia. J Clin Oncol. 2003;21(24):4642-9.

12. Tong WD, et al. ArrayTrack - supporting toxicogenomic research at the US Food and Drug Administration National Center for Toxicological Research. Environ Health Perspect. 2003;111(15):1819-26.

13. Jelinek J, et al. Conserved DNA methylation patterns in healthy blood cells and extensive changes in leukemia measured by a new quantitative technique. Epigenetics. 2012:7(12):1368-78.

14. Jelinek J, Madzo J. DREAM: a simple method for DNA methylation profiling by high-throughput sequencing. In: Li S, Zhang H, editors. Chronic myeloid leukemia: methods and protocols. Totowa: Humana Press Inc; 2016. p. 111-27.

15. van Buuren S, Groothuis-Oudshoorn K. Mice: multivariate imputation by chained equations in R. J Stat Softw. 2011;45(3):1-67.

16. R Development Core Team. R: a language and environment for statistical computing. Vienna: R Foundation for Statistical Computing; 2017.

17. Issa JP. CpG island methylator phenotype in cancer. Nat Rev Cancer. 2004; 4(12):988-93.

18. Fullerton JN, O'Brien AJ, Gilroy DW. Lipid mediators in immune dysfunction after severe inflammation. Trends Immunol. 2014;35(1):12-21.

19. Fandy $T E$, et al. Early epigenetic changes and DNA damage do not predict clinical response in an overlapping schedule of 5-azacytidine and entinostat in patients with myeloid malignancies. Blood. 2009;114(13):2764-73.

20. Jones PA, Issa JP, Baylin S. Targeting the cancer epigenome for therapy. Nat Rev Genet. 2016;17(10):630-41.

21. Figueroa ME, et al. Leukemic IDH1 and IDH2 mutations result in a hypermethylation phenotype, disrupt TET2 function, and impair hematopoietic differentiation. Cancer Cell. 2010;18(6):553-67.

22. Amatangelo MD, et al. Enasidenib induces acute myeloid leukemia cell differentiation to promote clinical response. Blood. 2017;130(6):732-41.

23. Welch JS, et al. TP53 and decitabine in acute myeloid leukemia and myelodysplastic syndromes. N Engl J Med. 2016;375(21):2023-36.

24. Montalban- B, Takahashi K, Garcia-Manero G. Decitabine in TP53-mutated AML. N Engl J Med. 2017;376(8):796-7.

25. Wiehle $\mathrm{L}$, et al. DNA (de) methylation in embryonic stem cells controls CTCF-dependent chromatin boundaries. Genome Res. 2019;29(5):750-61.

26. Hayette $S$, et al. High DNA methyltransferase DNMT3B levels: a poor prognostic marker in acute myeloid leukemia. PLoS One. 2012;7(12):e51527.

27. Lamba JK, et al. Integrated epigenetic and genetic analysis identifies markers of prognostic significance in pediatric acute myeloid leukemia. Oncotarget. 2018;9(42):26711-23.

28. Niederwieser $\mathrm{C}$, et al. Prognostic and biologic significance of DNMT3B expression in older patients with cytogenetically normal primary acute myeloid leukemia. Leukemia. 2015:29(3):567-75.

29. Elliott EN, Sheaffer KL, Kaestner KH. The 'de novo' DNA methyltransferase Dnmt3b compensates the Dnmt1-deficient intestinal epithelium.Elife. 2016; 5:e12975.

30. Hur EH, et al. Establishment and characterization of hypomethylating agent-resistant cell lines, MOLM/AZA-1 and MOLM/DEC-5. Oncotarget. 2017:8(7):11748-62.

31. Tsujioka T, et al. Effects of DNA methyltransferase inhibitors (DNMTIs) on MDS-derived cell lines. Exp Hematol. 2013;41(2):189-97.

32. Simo-Riudalbas L, Melo SA, Esteller M. DNMT3B gene amplification predicts resistance to DNA demethylating drugs. Genes Chromosomes Cancer. 2011; 50(7):527-34.

33. Jabbour $\mathrm{E}$, et al. Prognostic factors associated with disease progression and overall survival in patients with myelodysplastic syndromes treated with decitabine. Clin Lymphoma Myeloma Leuk. 2013;13(2):131-8.

34. Neubauer $A$, et al. Patients with acute myeloid leukemia and RAS mutations benefit most from postremission high-dose cytarabine: a cancer and leukemia group B study. J Clin Oncol. 2008;26(28):4603-9.

\section{Publisher's Note}

Springer Nature remains neutral with regard to jurisdictional claims in published maps and institutional affiliations. 